\title{
Review Article \\ High Fasting Plasma Glucose during Early Pregnancy: A Review about Early Gestational Diabetes Mellitus
}

\author{
E. Cosson, ${ }^{1,2}$ L. Carbillon, ${ }^{3}$ and P. Valensi ${ }^{1}$ \\ ${ }^{1}$ Department of Endocrinology-Diabetology-Nutrition, AP-HP, Jean Verdier Hospital, Paris 13 University, Sorbonne Paris Cité, \\ CRNH-IdF, CINFO, Bondy, France \\ ${ }^{2}$ Sorbonne Paris Cité, UMR U1153 Inserm/U1125 Inra/Cnam/Université Paris 13, Bobigny, France \\ ${ }^{3}$ Department of Gynecology-Obstetrics, AP-HP, Jean Verdier Hospital, Paris 13 University, Sorbonne Paris Cité, Bondy, France
}

Correspondence should be addressed to E. Cosson; emmanuel.cosson@aphp.fr

Received 27 July 2017; Accepted 19 September 2017; Published 18 October 2017

Academic Editor: Patrizio Tatti

Copyright (c) 2017 E. Cosson et al. This is an open access article distributed under the Creative Commons Attribution License, which permits unrestricted use, distribution, and reproduction in any medium, provided the original work is properly cited.

Fasting plasma glucose (FPG) is nowadays routinely measured during early pregnancy to detect preexisting diabetes (FPG $\geq 7 \mathrm{mmol} / \mathrm{L}$ ). This screening has concomitantly led to identify early intermediate hyperglycemia, defined as FPG in the 5.1 to $6.9 \mathrm{mmol} / \mathrm{L}$ range, also early gestational diabetes mellitus (eGDM). Early FPG has been associated with poor pregnancy outcomes, but the recommendation by the IADPSG to refer women with eGDM for immediate management is more pragmatic than evidence based. Although eGDM is characterized by insulin resistance and associated with classical risk factors for type 2 diabetes and incident diabetes after delivery, it is not necessarily associated with preexisting prediabetes. FPG $\geq 5.1 \mathrm{mmol} / \mathrm{L}$ in early pregnancy is actually poorly predictive of gestational diabetes mellitus diagnosed after 24 weeks of gestation. An alternative threshold should be determined but may vary according to ethnicity, gestational age, and body mass index. Finally, observational data suggest that early management of intermediate hyperglycemia may improve prognosis, through reduced gestational weight gain and potential early introduction of hypoglycemic agents. Considering all these issues, we suggest an algorithm for the management of eGDM based on early FPG levels that would be measured in case of risk factors. Nevertheless, interventional randomized trials are still missing.

\section{Introduction}

Gestational diabetes mellitus (GDM) was historically defined as "any degree of glucose intolerance with onset or first recognition during pregnancy," whatever the treatment course and postpartum evolution [1]. Chronic insulin resistance in the second half of pregnancy is a central component of the pathophysiology of GDM [2, 3]. Diagnostic criteria were therefore established for 24-28 weeks of gestation (WG) including both glucose values during an oral glucose tolerance test (OGTT) and incident events [1]. The International Association of Diabetes Pregnancy Study Group (IADPSG) proposed the following diagnostic criteria: fasting plasma glucose (FPG) value $\geq 5.1 \mathrm{mmol} / \mathrm{L}$ and/or 1 -hour glucose value $\geq 10.0 \mathrm{~m}$ $\mathrm{mol} / \mathrm{L}$ and/or 2-hour glucose value $\geq 8.5 \mathrm{mmol} / \mathrm{L}$. Although the National Institute for Health and Care Excellence (NICE) did not (http://www.nice.org.uk/guidance/ng3/evidence), these diagnostic criteria have been adopted worldwide, for example, by the American Diabetes Association (ADA) [4], the American Association of Clinical Endocrinologists [5], the Société Francophone du Diabète and the Collège National des Gynécologues et Obstétriciens Français [6], the International Federation of Gynecology and Obstetrics (FIGO) [7], and the Italian National Institute of Health (ISS) (http://www.salute.gov.it). The IADPSG criteria for the diagnosis of GDM are therefore now commonly called the 2013 WHO criteria for GDM (http://apps.who.int/iris/bitstream/ 10665/85975/1/WHO_NMH_MND_13.2_eng.pdf).

As the proportion of patients with unknown type 2 diabetes has been increasing, a new category of glucose disorder was introduced with the IADPSG recommendations. Women are considered to have overt diabetes [1] or diabetes in pregnancy (DIP (WHO criteria)) if their plasma glucose values are above the thresholds defining diabetes outside of 
pregnancy: FPG value $\geq 7 \mathrm{mmol} / \mathrm{L}$ and/or 2-hour glucose value $\geq 11.1 \mathrm{mmol} / \mathrm{L}$ and/or $\mathrm{HbA} 1 \mathrm{c} \geq 6.5 \%$. Preconceptional diagnosis of diabetes or prediabetes would actually be useful to optimize glucose levels prior to conception but is rarely performed. On another hand, waiting for $24 \mathrm{WG}$ to diagnose DIP would delay care in an unacceptable way and potentially lead to severe obstetric complications, as obstetric outcomes are quite similar for undiagnosed type 2 and type 1 diabetes [8]. Furthermore, the prevalence of fetal malformations was recently reported to be higher in women with DIP compared to women with GDM [9]. The incidence of other outcomes was similar in both groups [9].

Concomitantly, the IADPSG recommended using a FPG range $5.1-6.9 \mathrm{mmol} / \mathrm{L}$ before $24 \mathrm{WG}$ to define early GDM (eGDM). Measurement was recommended at the first prenatal visit, or later if undone, especially in high-risk women [1]. The threshold of $5.1 \mathrm{mmol} / \mathrm{L}$ is arbitrary and was chosen as the same value as after 24 WG. Actually, FPG was described to be quite stable during pregnancy [10]. In this study, FPG levels were similar in the same women when measured at 17 WG then at 32 WG [10], despite an increase in insulin secretion and a propensity for earlier hypoglycemia during fasting as described a long time ago [11]. The IADPSG recommends that women with eGDM should be referred for immediate care, even if the level of proof for this recommendation is very low regarding to prognosis $[12,13]$. Alternatively, some guidelines do not recommend to treat immediately women with high FPG levels in early pregnancy, such as the ADA [4]. Other advice for patients who have an early FPG between 5.6 and $6.9 \mathrm{mmol} / \mathrm{L}$ is to perform an OGTT at 16-18 WG and to monitor and treat abnormal results at this time (http://www.salute.gov.it).

\section{Methods}

References for this review were identified through searches of PubMed for articles published until March 2017, by use of the terms "early gestational diabetes mellitus" and "fasting plasma glucose and pregnancy." English and French articles resulting from these searches and relevant references cited in those articles were reviewed. We discuss here the prevalence, risk factors, and metabolic characteristics of eGDM, as well as its prognosis.

\section{Results}

3.1. Bibliography. Our search in Medline suggested 1800 references between January 2010 and March 2017. Of these articles, we selected and analysed around 100 articles. We additionally analysed around 20 articles that were cited in these papers. We also found 5 relevant ongoing studies in clinicaltrials.gov. We finally selected 49 references.

\subsection{The Burden of eGDM}

3.2.1. Diagnosis with Early FPG Measurement. A FPG level $\geq 5.0 \mathrm{mmol} / \mathrm{L}$ was reported in $11.9 \%$ of pregnant women during the first trimester of pregnancy (mean $9 \mathrm{WG}$ ) in Israel [14], where universal screening for FPG has been recommended at the first prenatal care visit. This prevalence was close to the prevalence of FPG above $5.1 \mathrm{mmol} / \mathrm{L}$ that was reported at the first prenatal visit in a multicenter study in China [15] and in one center in Italy [16] (11.4\% and $7.2 \%$, resp.), noting that almost all women were tested in these studies.

In France, where selective screening and using IADPSG criteria is recommended, data from the French National Interscheme Health Insurance Information System (SNIIRAM) showed that only $2.3 \%$ of 788,494 pregnant women in 2013 without known diabetes were treated for a dysglycemia diagnosed before 22 WG [17]. These women corresponded to $26.9 \%$ of all women with dysglycemia during their pregnancy. The French criteria for selective screening are maternal age $\geq 35$ years, body mass index $(\mathrm{BMI}) \geq 25 \mathrm{~kg} / \mathrm{m}^{2}$, history of diabetes in a first-degree relative, personal history of GDM, or having giving birth to a child with fetal macrosomia [6]. In the United States, implementing early screening nearly doubled the incidence of GDM as compared with a previous standard two-step approach [18].

3.2.2. Diagnosis with Early OGTT. A high prevalence of eGDM has also been reported when OGTT is performed in early pregnancy in selected populations with risk factors. The prevalence was $23.4 \%$ in pregnant women with a BMI at or above $29.0 \mathrm{~kg} / \mathrm{m}^{2}$ in early pregnancy as part of the enrollment into the DALI (Vitamin D And Lifestyle Intervention for GDM prevention) pilot and lifestyle Pan-European multicenter trials [19]. Additionally, the prevalence of eGDM in high-risk populations was recently reported to be $48.8 \%$ in Italy [20] and 27.3\% in Australia [21].

To conclude this part, intermediate FPG levels in early pregnancy, also known as eGDM, have become a very common issue.

3.3. Risk Factors for Hyperglycemia in Early Pregnancy. Figure 1 shows that FPG $\geq 5.1 \mathrm{mmol} / \mathrm{L}$ during early pregnancy is more prevalent with aging $[16,19,22]$, higher BMI $[16,19,22]$, a family history of diabetes $[19,22]$, a personal history of GDM $[19,22]$ or newborn with macrosomia [19], and multiparity [23].

Studies using other definition for eGDM found similar risk factors [21, 23-26]. However, all those results should be interpreted with caution as screening is usually performed in already high-risk subjects.

3.4. Metabolic Characteristics of Women with eGDM. Bozkurt et al. have evaluated pathophysiological characteristics of pregnant women diagnosed with GDM according to IADPSG criteria [22]. Unlike patients with late (regular) GDM or normal glucose tolerant women, subjects with eGDM exhibited decreased insulin sensitivity, with lower oral glucose insulin sensitivity index and quantitative insulin sensitivity check index. The subgroups also differed in BMI, with significantly higher levels in patients with eGDM compared to subjects with late GDM and those with normal glucose tolerance $\left(31.7 \pm 6.4,27.7 \pm 4.4\right.$, and $27.3 \pm 5.6 \mathrm{~kg} / \mathrm{m}^{2}$, resp., $p<0.001)$. However, differences in estimated insulin sensitivity remained significant after adjustment for BMI, 


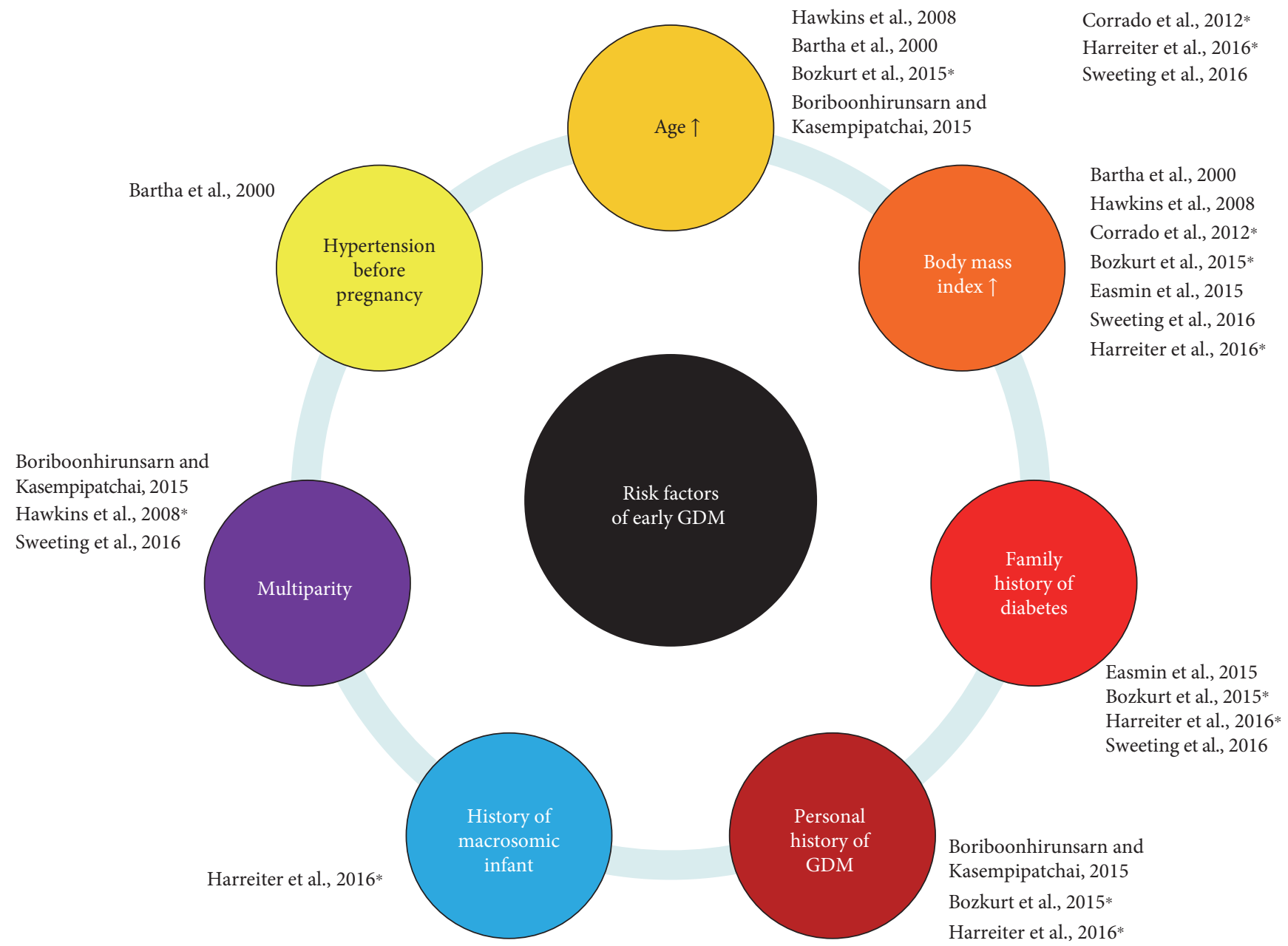

FIGURE 1: Risk factors for early gestational diabetes mellitus. GDM: gestational diabetes mellitus. * identifies references where early GDM is defined according to the IADPSG definition, that is, fasting plasma glucose value $\geq 5.1 \mathrm{mmol} / \mathrm{L}$.

age, and history of GDM. In this study, subjects with either early or late GDM showed impairments in $\beta$-cell function (insulinogenic index) as compared with women without GDM [22].

In another study including women with BMI at or above $29 \mathrm{~kg} / \mathrm{m}^{2}$ who were screened in early pregnancy using a $75 \mathrm{~g}$ OGTT, various indexes of insulin sensitivity (oral glucose insulin sensitivity index, quantitative insulin sensitivity check index, and homeostasis model assessment of insulin resistance (HOMA-IR)) and secretion (Stumvoll first and second phases) decreased progressively in women with normal glucose tolerance, eGDM, and DIP [19]. Differences persisted after adjustment for age, pregestational BMI, gestational week, and fetal gender [19].

3.5. What Does eGDM Outside Pregnancy Mean? Mills et al. [27] have shown that there is a physiological reduction in FPG concentration in normal pregnancy. In their cohort of 361 healthy pregnant women, they showed that FPG levels decrease with advancing pregnancy with a plateau occurring around 10-20 WG [27]. Thereafter, insulin resistance increases and "late" GDM may occur [28]. As shown and explained in Figure 2 and as it was previously suggested [29], high FPG during early pregnancy could indicate unknown prediabetes (if eGDM) or diabetes (if DIP) before pregnancy as insulin resistance remains present in both cases. Although glycemic status before pregnancy is usually unknown, we may use several indicators, such as markers for glycemic exposure before pregnancy, early postdelivery glycemic status (which is usually considered to reflect glycemic status before pregnancy), and incidence of type 2 diabetes postpartum.

3.5.1. Marker for Long-Term Glycemic Exposure at the Time of eGDM Diagnosis. Skin autofluorescence, a measurement of cutaneous advanced glycation end products, can be used as a screening method in detecting unknown diabetes. Maury et al. have suggested that skin autofluorescence was a marker of metabolic memory in pregnant women. During pregnancy, forearm skin autofluorescence at 24-30 WG was reported to gradually decrease from patients with previous diabetes, to women with GDM and previous hyperglycemia, to those with GDM without previous hyperglycemia, and finally to normal subjects without diabetes or GDM [30]. In 


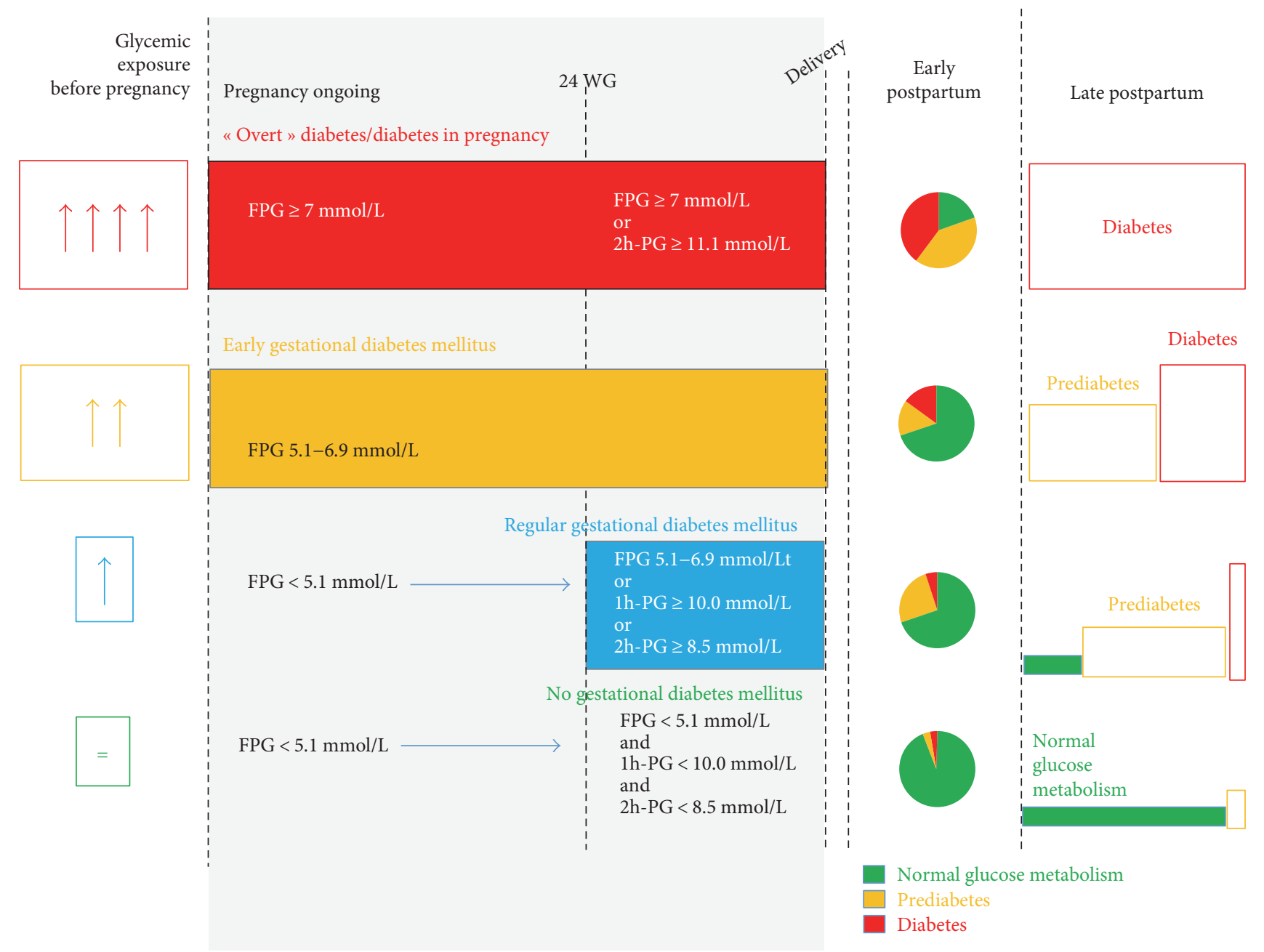

FIGURE 2: Does eGDM mean preexisting prediabetes? The hypothesis is when hyperglycemia has been present (but unknown) before pregnancy, then fasting plasma glucose (FPG) is already increased during early pregnancy while insulin resistance increases after 24 weeks of gestation (WG). Accordingly, oral glucose tolerance will reveal dysglycemia in early postpartum. 1h-PG and 2h-PG: plasma glucose 1 and 2 hours after $75 \mathrm{~g}$ oral glucose tolerance test; FPG: fasting plasma glucose; WG: weeks of gestation.

that study, previous hyperglycemia was defined as previous GDM or having given birth to a macrosomic infant or GDM diagnosed before 24 WG (eGDM) [30].

3.5.2. Data Based on OGTT in the Immediate Postpartum. Sweeting et al. have recently reported the results of postpartum OGTT according to the time of GDM diagnosis [21]. In this study, women were tested between 2001 and 2011, with early screening performed in women considered at high risk for GDM. The repartition of normal plasma glucose values, impaired glucose tolerance, and diabetes during OGTT performed three months postpartum significantly differed according to eGDM $<12 \mathrm{WG}$ (normal glucose tolerance $79 \%$, impaired glucose tolerance $11 \%$, and diabetes $11 \%)$, GDM 12-23 WG (71, 24, and 5\%, resp.), and $\mathrm{GDM}>24 \mathrm{WG}(85,14$, and 1\%, resp.). However, the proportion of dysglycemia was only $22 \%$ in women with eGDM $<12$ WG, suggesting that eGDM is not a good marker of preexisting dysglycemia. However, the implementation of a lifestyle change program during pregnancy and postpartum might partially account for this result.

3.5.3. Postpartum Development of Type 2 Diabetes. The presence of eGDM could also identify women with an increased risk to later develop type 2 diabetes. In a systematic review including 8 studies and 4026 women with GDM defined with numerous criteria, women with eGDM had a twofold increased risk of incident type 2 diabetes 6 weeks to 20 years after delivery compared to subjects with "late" GDM (relative risk (RR) 2.13 (95\% CI 1.52-3.56)) [31]. IADPSG-diagnosed eGDM has also been reported to be a significant predictor of progression to abnormal glucose tolerance up to 5 years postpartum. In this study, the earlier the GDM was diagnosed, the higher was the rate of dysglycemia up to 5 years postpartum [32].

To conclude at this step, although eGDM is associated with a profile comparable to metabolic syndrome, with higher insulin resistance than in "regular GDM," 
it has not been consistently associated with prediabetes or diabetes during early postpartum. However, it is associated with more incident type 2 diabetes than regular GDM (Figure 2). Overall, this suggests that eGDM should be considered as a new intermediate entity between normal glucose metabolism and prediabetes/diabetes outside pregnancy.

\subsection{The Meaning of an Early High FPG through Pregnancy}

3.6.1. Persistence through Pregnancy. eGDM was initially supposed to be persistent through pregnancy. Therefore, it was hypothesized that early care for eGDM would improve prognosis and that was the reason why FPG in the range 5.1-6.9 mmol/L was recommended to diagnose GDM at any time during pregnancy [1]. However, data from Italy [16] and China [15] have challenged this recommendation. Actually, at least $50 \%$ of the women with eGDM have no GDM after $24 \mathrm{WG}$, despite the absence of specific care. In the Italian publication, $55 \%$ of women with $\mathrm{FPG} \geq 5.1 \mathrm{mmol} / \mathrm{L}$ during early pregnancy had a normal OGTT after $24 \mathrm{WG}$ [16]; in the Chinese study, less than one-third of the women still had a FPG $\geq 5.1 \mathrm{mmol} / \mathrm{L}$ between the first prenatal visit and 24-28 WG [15].

3.6.2. Does Early FPG Predict GDM after 24 WG? Smirnakis et al. showed in a prospective study that women in whom GDM was diagnosed at 24-28 WG (using the O'Sullivan and Mahan criteria of the American Diabetes Association) demonstrated higher levels of FPG (4.8 \pm 0.6 versus 4.4 $\pm 0.4 \mathrm{mmol} / \mathrm{L}, p<0.05)$ and HOMA-IR at $17 \mathrm{WG}$ compared to women who had normoglycemic pregnancies [33]. RiskinMashia et al. have also shown that first-trimester FPG levels were positively associated with the risk of GDM after 24 $\mathrm{WG}$, especially when values were $\geq 5.0 \mathrm{mmol} / \mathrm{L}$ [14]. In another study including overweight women at very high risk for GDM, the prevalence of IADPSG-defined GDM after 24 WG was $53 \%$ in those with early FPG $\geq 4.9 \mathrm{mmol} / \mathrm{L}$ whereas the prevalences were 15,12 , and $20 \%$ in those with early $\mathrm{FPG} \leq 4.4,4.41-4.6$, and $4.61-4.89 \mathrm{mmol} / \mathrm{L}$, respectively [34]. Therefore, FPG in early pregnancy can be considered as a tool to select the women at risk for GDM after $24 \mathrm{WG}$. For example, the sensitivity, specificity, positive, and negative predictive values of FPG $>4.4 \mathrm{mmol} / \mathrm{L}$ in early pregnancy to predict IADPSG-defined GDM in an Italian population were $80 \%, 66 \%, 77 \%$, and $96 \%$, respectively [35]. Therefore, using the recursive portioning and amalgamation method, it was suggested to consider a low FPG to avoid OGTT after $24 \mathrm{WG}$ because of its high negative predictive value [35]. Actually, the negative predictive value was not so high in two other studies. First, in a Chinese population, the sensitivity, specificity, positive, and negative predictive values of $\mathrm{FPG}>4.4 \mathrm{mmol} / \mathrm{L}$ to predict IADPSG-defined GDM were $78 \%, 38 \%, 21 \%$, and $89 \%$, respectively [15]. Second, in overweight women at very high risk of GDM, the negative predictive value of early $\mathrm{FPG} \leq 4.4 \mathrm{mmol} / \mathrm{L}$ was only $85 \%$ [34].

Additionally, FPG concentration at first antenatal visit in 2284 women in China was higher in those who later developed GDM than in those who did not. However, early FPG was associated with late GDM when measured between 12 and 16 WG, 16-20 WG and 20-24 WG, but not before 12 WG. This suggested that the relationship between FPG and late GDM appeared during the second trimester [36].

\subsection{Should We Consider Alternatives to FPG Measurement in Early Pregnancy}

3.7.1. The Limits of Measuring FPG in Early Pregnancy. Analyzing FPG level is actually complex. First, FPG decreases with increasing gestational age [15]. For example, in a Chinese population [15], median FPG was $4.95 \mathrm{mmol} / \mathrm{L}$ at $4-6 \mathrm{WG}$, 4.70 at $10-12 \mathrm{WG}$, and 4.53 at $14-16 \mathrm{WG}$ and was the lowest at $4.38 \mathrm{mmol} / \mathrm{L}$ at $20-24 \mathrm{WG}$.

Second, based on the receiving operating curves and area under the curve measurements, in another Chinese population, the optimal FPG cut-off values to predict GDM after 24 WG were different according to BMI categorization groups [37]. The FPG cut-off value was $4.77 \mathrm{mmol} / \mathrm{L}$ in prepregnancy underweight women, $4.92 \mathrm{mmol} / \mathrm{L}$ in prepregnancy normal weight women, $5.00 \mathrm{mmol} / \mathrm{L}$ in prepregnancy overweight women, and $5.05 \mathrm{mmol} / \mathrm{L}$ in prepregnancy obese women [37].

Overall, the threshold for FPG could be different according to WG and to BMI level. There is a need to investigate the best threshold for early FPG and the association with late GDM or poor pregnancy outcomes. The concordances of early OGTT, at 12-15 WG [38] or at 18-20 WG [39], are going to be compared to OGTT results at 24-28 WG in two studies, but no data are currently available yet.

3.7.2. OGTT. OGTT could be more sensitive than FPG alone to diagnose GDM during early pregnancy. For example, in pregnant women with obesity at 15.2 $\pm 3.0 \mathrm{WG}$ and using IADPSG criteria, 1- and 2-hour glucose values led to diagnose $21.5 \%$ additional GDM as compared to FPG value during the OGTT [19]. However, data on 1- and 2-hour OGTT glucose values are rare in early pregnancy. Using IADPSG criteria is not evidence based in early pregnancy. Especially, thresholds that are used after $24 \mathrm{WG}$ are too high for early pregnancy [10]. OGTT is time-consuming, inconvenient, and uncomfortable, inducing nausea and vomiting in some patients. Therefore, some women might refuse to repeat OGTT after $24 \mathrm{GW}$.

3.7.3. HbA1c Measurement. HbA1c measurement also warrants further evaluation [13]. HbAlc $\geq 5.9 \%$ has been reported to identify all cases of DIP and to be associated with poor pregnancy outcomes, including congenital anomalies, preeclampsia, shoulder dystocia, and perinatal deaths [40]. The association with poor outcomes appears to be independent of later GDM diagnosis [41]. However, HbA1c may vary with pregnancy hemodilution [42] and with the presence of hemoglobinopathy and/or anemia.

To conclude this part, FPG $>5.1 \mathrm{mmol} / \mathrm{L}$ persists in less than one-half of untreated women and we should define an alternative FPG threshold to define eGDM. However, it may differ according to BMI and WG. 


\subsection{Prognosis Related to Hyperglycemia in Early Pregnancy (Table 1)}

3.8.1. Poor Pregnancy Prognosis Related to FPG in Early Pregnancy. A poor prognosis of high FPG levels during the first trimester was reported, with an increased risk of adverse pregnancy outcomes, including macrosomia $[14,36]$ and cesarean section [14].

Among the 788,494 women who delivered in France in 2013, women with early onset of GDM were more likely to have need for cesarean section (odds ratio 1.10 (95\% confidence interval 1.05-1.15)) and large for gestational age infants $(1.18(1.12-1.24))$ than women diagnosed between 22 and $30 \mathrm{WG}$ [17].

3.8.2. Poor Prognosis Associated with eGDM Not Defined with FPG. Bartha et al. [24] have compared complications associated with GDM diagnosed in early (mainly during the first trimester) or late pregnancy. eGDM screening was performed only in case of risk factors, especially increased BMI, which was a bias as the presence of risk factors has been associated with a worse prognosis [43]. They reported that the group diagnosed earlier in pregnancy had higher rates of preeclampsia, neonatal hypoglycemia, and perinatal deaths and lower rate of oligohydramnios [24]. Preeclampsia, shoulder dystocia, macrosomia, and hyperbilirubinemia were reported to be more frequent in women with diet-treated GDM diagnosed before than after $24 \mathrm{WG}$, even after adjustment for maternal age, ethnicity, parity, weight, and blood glucose control [23]. Obstetric outcomes were also compared in early onset and late onset GDM in Bangladesh and showed a poorer prognosis associated with eGDM, including more preeclampsia, neonatal admission in intensive care unit, and neonatal hypoglycemia [26].

When using the Australasian Diabetes in Pregnancy Society diagnostic criteria in a large Australian multiethnic cohort of women considered at high risk for GDM, eGDM was also associated with poorer pregnancy outcomes [21]. In this study, the eGDM cohort was a preselected high-risk group and patients with eGDM diagnosed before 12 WG had an intermediate risk profile, standing between the risk of subjects with preexisting type 2 diabetes and the risk of patients diagnosed with eGDM between 12 and 23 WG. The outcomes in women who were diagnosed with GDM before $12 \mathrm{WG}$ were quite comparable to those observed in subjects with preexisting diabetes despite early testing and current best practice treatment [21].

Further research is currently necessary to evaluate the effects of early metabolic changes on short- and long-term outcomes for the mother and the child and of the potential consequences on generational transmission of metabolic diseases.

\subsection{Does the Treatment of Hyperglycemia in Early Pregnancy Improve Prognosis? (Table 1)}

3.9.1. Argumentation from Retrospective Studies Comparing Women Cared for Early or Regular GDM. Early detection and treatment of women at high risk of eGDM might improve pregnancy outcomes: several studies have shown a similar prognosis in early and late GDM with treatment. For example, an Indian team assessed the merits of care given to women in whom GDM was diagnosed in different WG in order to find out the ideal period of screening in women with history of high-risk pregnancies. The babies born to GDM women diagnosed before $12 \mathrm{WG}$ had a lower birth weight than the ones born to GDM women diagnosed after 30 WG. The authors concluded therefore that screening in the first trimester of pregnancy and institution of therapy was advisable in women with high-risk pregnancies [44]. In Thailand, the incidence of pregnancy complications was similar in women diagnosed with GDM early and late in pregnancy [25]. In this study, gestational weight gain and glycemic control, but not the time of diagnosis, were independently associated with macrosomia in women with GDM [25]. The authors suggested that the higher frequency of insulin treatment, as consistently reported [17, 18, 21, 24-26, 45], and the lower gestational weight gain $[21,24,25]$ in women with eGDM compared to women with late GDM might explain a similar prognosis in both groups in these studies. However, although insulin initiation was also earlier in eGDM than in late GDM, with a higher maximum daily insulin dose in the study by Sweeting et al., the prognosis was worse in women with eGDM [46].

3.9.2. Argumentation from Retrospective Studies Comparing Strategies including Early Screening for Dysglycemia or Not. Alunni et al. compared two cohorts of pregnant women with GDM: those diagnosed via two-step screening (standard approach) versus those diagnosed via early screening diagnosis with additional screening after $24 \mathrm{WG}$ if early screening was negative. The second approach doubled the incidence of GDM, but there was no significant difference in neonatal outcomes [18].

Hong et al. analyzed a retrospective cohort of women with singleton pregnancies diagnosed with GDM who had indications for early screening, defined as the presence of obesity, or GDM or macrosomia in a prior pregnancy. Women were classified as having been screened early $(<20 \mathrm{WG})$ or routinely $(>24 \mathrm{WG})$. The decision of whether or not a patient had early screening for GDM was at the discretion of the managing provider. Early screening was not associated with significant reduction in the risk of cesarean, preeclampsia, macrosomia, or birth injury. The authors concluded that the utility of early GDM screening still required an evaluation [45].

3.9.3. Future Randomized Trials. Randomized controlled trials evaluating the benefit-cost balance for screening and treating less severe hyperglycemia than DIP in early pregnancy are mandatory [13]. We have found several ongoing trials. One will compare the prognosis of pregnancies of 202 women with first trimester hyperglycemia (defined with FPG or $\mathrm{HbAlc}$ ) when treatment begins before $15 \mathrm{WG}$ versus after 28 WG (clinicaltrials.gov NCT01926457). The Early Gestational Diabetes Screening in the Gravid Obese Woman (EGGO, clinicaltrials.gov NCT01864564) and the Randomization of Early Diabetes Screening Among Obese Pregnant 


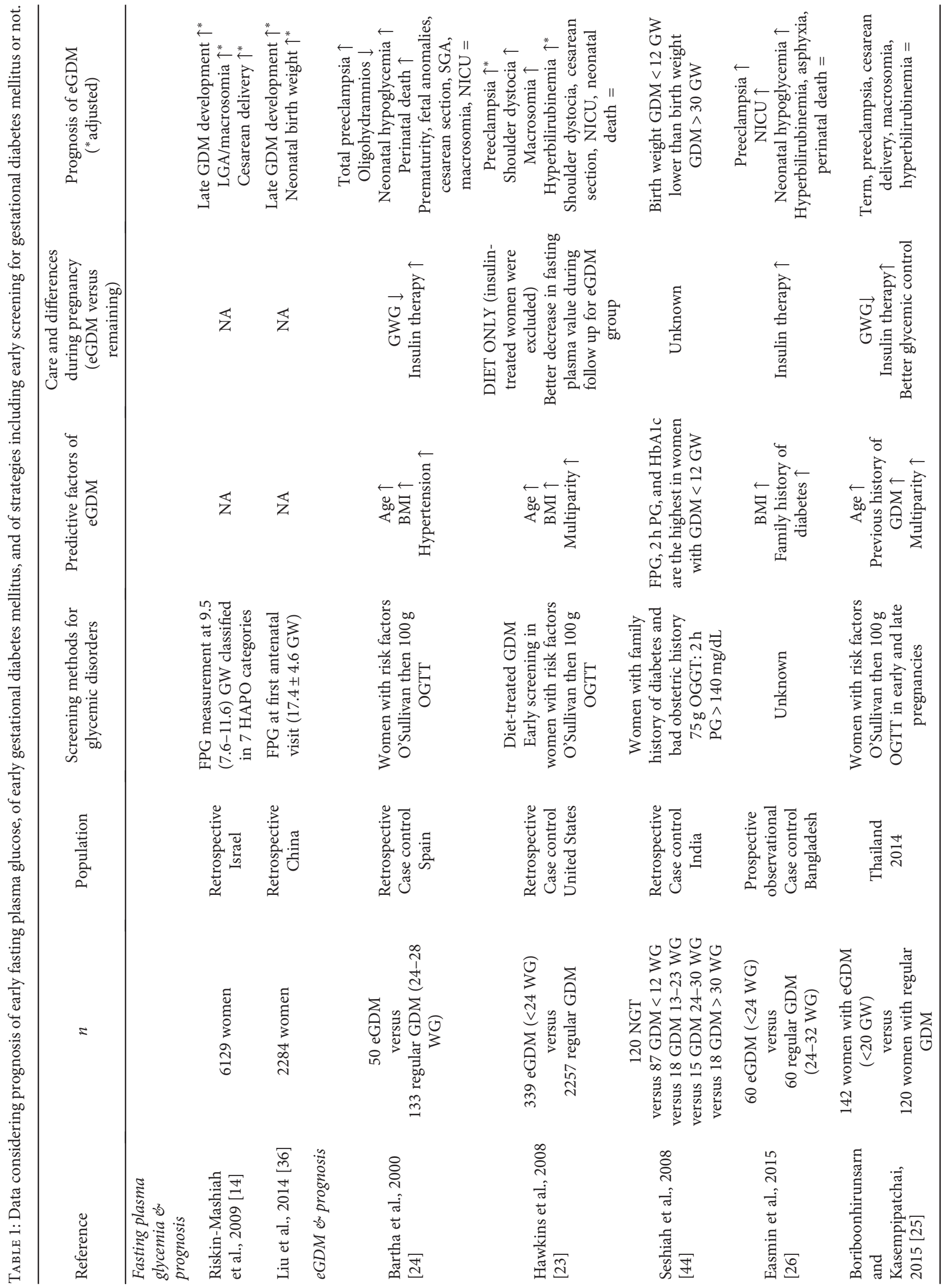




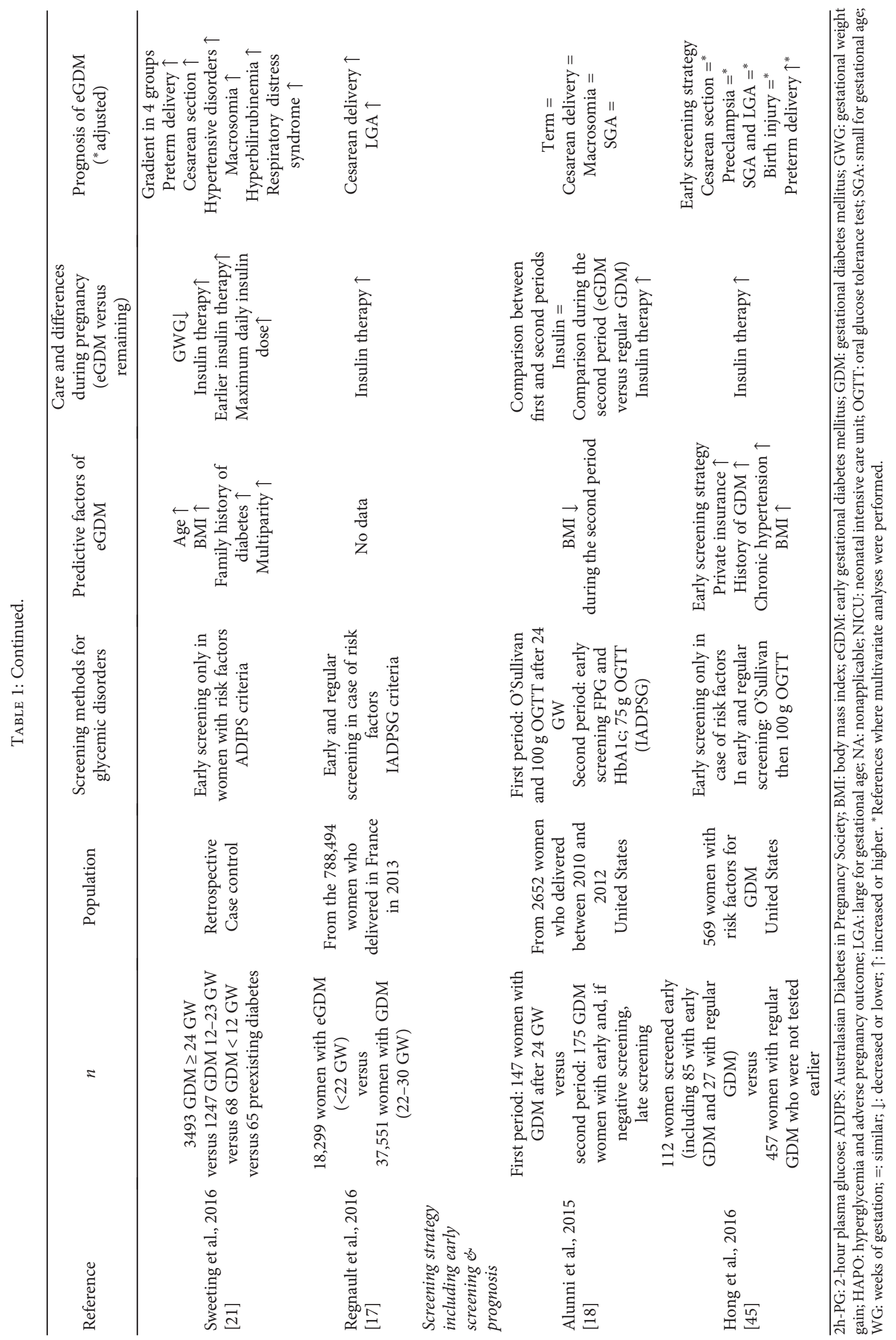




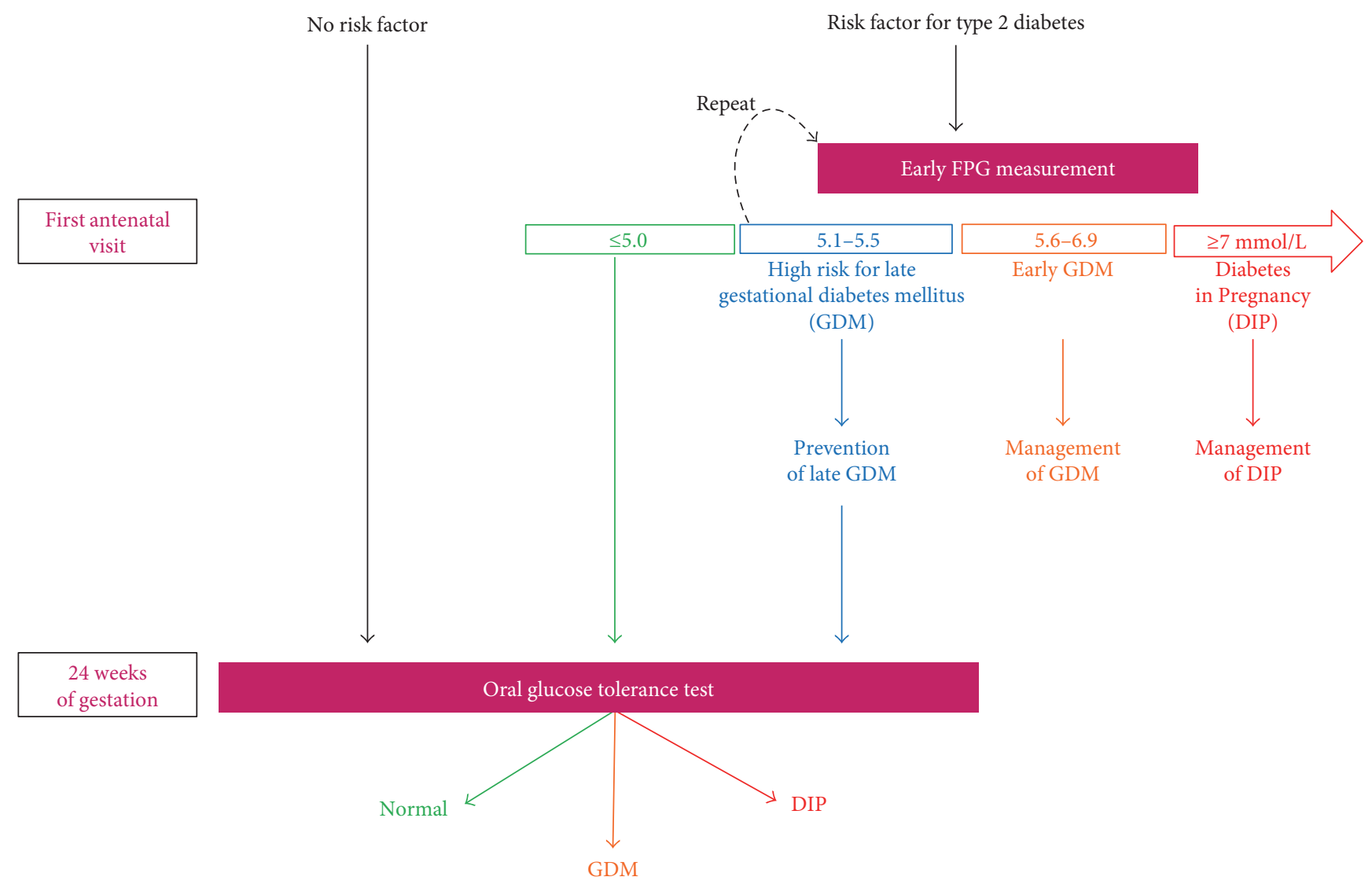

FIgURE 3: Proposals for a management algorithm according to the presence of risk factors and screening for dysglycemia during pregnancy. DIP: diabetes in pregnancy; FPG: fasting plasma glucose; GDM: gestational diabetes mellitus.

Women (REDSOAP, clinicaltrials.gov NCT03116009) studies will include 1150 and 600 obese women, respectively. They will compare the prognosis associated with screening strategies including or not a screening for dysglycemia during early pregnancy. Finally, the Early Diagnosis of Gestational Diabetes Mellitus Study (EDoGDM) will randomize 600 low-risk pregnant women with an OGTT performed at 18 to $20 \mathrm{WG}$ versus 24 and $28 \mathrm{WG}$ and will compare prognosis in both groups ([39], clinicaltrials.gov NCT02740283).

To conclude this part, high FPG during early pregnancy is associated with a poor prognosis, which might be improved with immediate care through diet and insulin therapy if necessary. However, the results of randomized trials are still lacking.

3.10. What Could Be Proposed in Clinical Practice at the Current Time? We propose the following algorithm of management according to early FPG (Figure 3 ). This proposal has not been validated by an expert consensus and should obviously not be considered as validated recommendations. The increasing number of subjects with undiagnosed type 2 diabetes mellitus before pregnancy justifies the screening of women with risk factors for preexisting diabetes by the first antenatal visit. We currently suggest to keep diagnosing DIP when FPG is $7.0 \mathrm{mmol} / \mathrm{L}$ or above. Actually, the incidence of macrosomia, preeclampsia, and neonatal hypoglycemia has been reported to be similar in treated women with DIP or GDM [9]. As eGDM is also associated with classical risk factors for type 2 diabetes, we suggest, for feasibility reasons, to screen early in pregnancy only the subjects considered to be at high risk. We suggest to test subjects with characteristics predisposing to type 2 diabetes according to the local frequency of abnormal glucose metabolism and according to local guidelines.

The current recommended FPG threshold $(5.1 \mathrm{mmol} / \mathrm{L})$ to define eGDM is an issue, as FPG depends on gestational age, ethnicity, and BMI categories [13, 15, 37]. We suggest to refer for care any subject with a FPG between 5.6 and $6.9 \mathrm{mmol} / \mathrm{L}$ as the risk of giving birth to a child with macrosomia increases with higher FPG [14, 36, 47]. We acknowledge that a FPG threshold at $5.6 \mathrm{mmol} / \mathrm{L}$ is also arbitrary as the association between early FPG and pregnancy outcomes appears to be a continuum [48]. However, Zhu et al. [15] showed that the positive predictive value of an abnormal OGTT at 24-28 weeks was $60 \%$ from an early FPG $5.7 \mathrm{mmol} / \mathrm{L}$, and last but not the least, Riskin-Mashiah et al. [14] reported in 6129 women with early FPG that the risk of macrosomia and primary cesarean section almost tripled and doubled, respectively, from this same threshold. Accordingly and as shown in the FIGO report [7], FPG values between 5.6 and $6.9 \mathrm{mmol} / \mathrm{L}$ are considered as GDM in China, Latin America, and the UK.

Because women might have inadequately fasted before the first measurement and because the reproducibility of FPG is 
imperfect, we propose to recheck the level of FPG when it is between 5.1 and $5.5 \mathrm{mmol} / \mathrm{L}$ and to consider the lowest value among both FPG measurements. HbA1c measurement might be an alternative to the second FPG but warrants further evaluation [13]. If the level of FPG is confirmed to be between 5.1 and $5.5 \mathrm{mmol} / \mathrm{L}$, we suggest lifestyle changes (nutrition and exercise). Actually, the prevention of GDM seems more effective when started before 15 WG [49]. These women should be screened again, with OGTT, after 24 WG.

We think that all the remaining women, even those with a low early FPG level, should be screened after 24 WG. Actually, an early FPG $<4.4 \mathrm{mmol} / \mathrm{L}$ had a very high negative predictive value for late GDM in one study [35] but this was not confirmed in two other studies [15, 34]. Therefore, we do propose to keep a late screening in these women. Noteworthily, the screening after $24 \mathrm{WG}$ includes women without risk factor but we have previously reported that women with GDM, even without risk factor and with adequate management, have a poor prognosis [43].

\section{Conclusions}

Measuring FPG in early pregnancy appears to be crucial to diagnose DIP and treat this condition as early as possible. Intermediate FPG levels in early pregnancy, also known as eGDM, have become a frequent issue. eGDM appears to represent a new intermediate entity in the following continuum: normal glucose metabolism, late GDM, eGDM, DIP, and pregravidic diabetes. Indeed, women diagnosed with eGDM have more risk factors for prevalent type 2 diabetes compared to subjects diagnosed with GDM later in pregnancy. They also have more incident type 2 diabetes and are more insulin resistant. This might participate to a poorer prognosis. However, there are major remaining issues about this new entity. Indeed, (i) a FPG $>5.1 \mathrm{mmol} / \mathrm{L}$ has been reported to persist in less than one-half of untreated women and the definition of eGDM needs to be better determined and (ii) there is nowadays no clear evidence of the usefulness of its treatment.

\section{Abbreviations}

\begin{tabular}{|c|c|}
\hline ADA: & American Diabetes Association \\
\hline BMI: & Body mass index \\
\hline DIP: & Diabetes in pregnancy \\
\hline eGDM: & Early gestational diabetes mellitus \\
\hline FIGO: & $\begin{array}{l}\text { International Federation of Gynecology and } \\
\text { Obstetrics }\end{array}$ \\
\hline FPG: & Fasting plasma glucose \\
\hline GDM: & Gestational diabetes mellitus \\
\hline HOMA-IR: & $\begin{array}{l}\text { Homeostasis model assessment of insulin } \\
\text { resistance }\end{array}$ \\
\hline IADPSG: & $\begin{array}{l}\text { International Association of Diabetes Pregnancy } \\
\text { Study Group }\end{array}$ \\
\hline NICE: & $\begin{array}{l}\text { National Institute for Health and Care } \\
\text { Excellence }\end{array}$ \\
\hline OGTT: & Oral glucose tolerance test \\
\hline WG: & Weeks of gestation. \\
\hline
\end{tabular}

\section{Conflicts of Interest}

The authors declare that they have no conflicts of interest.

\section{Acknowledgments}

The authors thank Dr. Sylvie Picard for the English revision and useful advices about the manuscript.

\section{References}

[1] B. E. Metzger, S. G. Gabbe, B. Persson et al., "International Association of Diabetes and Pregnancy Study Groups recommendations on the diagnosis and classification of hyperglycemia in pregnancy," Diabetes Care, vol. 33, no. 3, pp. 676-682, 2010.

[2] P. M. Catalano, E. D. Tyzbir, R. R. Wolfe et al., "Carbohydrate metabolism during pregnancy in control subjects and women with gestational diabetes," The American Journal of Physiology, vol. 264, no. 1, Part 1, pp. E60-E67, 1993.

[3] L. A. Barbour, C. E. McCurdy, T. L. Hernandez, J. P. Kirwan, P. M. Catalano, and J. E. Friedman, "Cellular mechanisms for insulin resistance in normal pregnancy and gestational diabetes," Diabetes Care, vol. 30, Supplement 2, pp. S112-S119, 2007.

[4] American Diabetes Association, "Diagnosis and classification of diabetes mellitus," Diabetes Care, vol. 40, Supplement 1, pp. S11-S24, 2017.

[5] Y. Handelsman, J. I. Mechanick, L. Blonde et al., “American Association of Clinical Endocrinologists Medical Guidelines for Clinical Practice for developing a diabetes mellitus comprehensive care plan," Endocrine Practice, vol. 17, Supplement 2, pp. 1-53, 2011.

[6] Gestational diabetes, "Summary of expert consensus," Diabetes \& Metabolism, vol. 36, no. 6, Part 2, pp. 695-699, 2010.

[7] M. Hod, A. Kapur, D. A. Sacks et al., "The International Federation of Gynecology and Obstetrics (FIGO) initiative on gestational diabetes mellitus: a pragmatic guide for diagnosis, management, and care," International Journal of Gynaecology and Obstetrics, vol. 131, Supplement 3, pp. S173-S211, 2015.

[8] A. McGrogan, J. Snowball, and C. S. de Vries, "Pregnancy losses in women with type 1 or type 2 diabetes in the UK: an investigation using primary care records," Diabetic Medicine, vol. 31, no. 3, pp. 357-365, 2014.

[9] F. Corrado, B. Pintaudi, R. D'Anna, A. Santamaria, L. Giunta, and A. Di Benedetto, "Perinatal outcome in a Caucasian population with gestational diabetes and preexisting diabetes first diagnosed in pregnancy," Diabetes \& Metabolism, vol. 42, no. 2, pp. 122-125, 2016.

[10] C. D. Agardh, A. Aberg, and N. E. Norden, "Glucose levels and insulin secretion during a $75 \mathrm{~g}$ glucose challenge test in normal pregnancy," Journal of Internal Medicine, vol. 240, no. 5, pp. 303-309, 1996.

[11] B. E. Metzger, V. Ravnikar, R. A. Vileisis, and N. Freinkel, "'Accelerated starvation" and the skipped breakfast in late normal pregnancy," The Lancet, vol. 319, no. 8272, pp. 588592, 1982.

[12] A. M. Guedj, "When should screening be performed for gestational diabetes?," Diabetes \& Metabolism, vol. 36, no. 6, Part 2, pp. 652-657, 2010. 
[13] H. D. McIntyre, D. A. Sacks, L. A. Barbour et al., "Issues with the diagnosis and classification of hyperglycemia in early pregnancy," Diabetes Care, vol. 39, no. 1, pp. 53-54, 2016.

[14] S. Riskin-Mashiah, G. Younes, A. Damti, and R. Auslender, "First-trimester fasting hyperglycemia and adverse pregnancy outcomes," Diabetes Care, vol. 32, no. 9, pp. 1639-1643, 2009.

[15] W. W. Zhu, H. X. Yang, Y. M. Wei et al., "Evaluation of the value of fasting plasma glucose in the first prenatal visit to diagnose gestational diabetes mellitus in China," Diabetes Care, vol. 36, no. 3, pp. 586-590, 2013.

[16] F. Corrado, R. D'Anna, M. L. Cannata, M. L. Interdonato, B. Pintaudi, and A. Di Benedetto, "Correspondence between first-trimester fasting glycaemia, and oral glucose tolerance test in gestational diabetes diagnosis," Diabetes \& Metabolism, vol. 38, no. 5, pp. 458-461, 2012.

[17] N. Regnault, E. Moutengou, and S. Fosse-Edorh, "Differential materno-fetal outcomes according to the onset date of gestational diabetes mellitus: data from 788,494 deliveries in France in 2013," EASD European Diabetes Epidemiology Group congress, 2016.

[18] M. L. Alunni, H. A. Roeder, T. R. Moore, and G. A. Ramos, "First trimester gestational diabetes screening - change in incidence and pharmacotherapy need," Diabetes Research and Clinical Practice, vol. 109, no. 1, pp. 135-140, 2015.

[19] J. Harreiter, D. Simmons, G. Desoye et al., "IADPSG and WHO 2013 gestational diabetes mellitus criteria identify obese women with marked insulin resistance in early pregnancy," Diabetes Care, vol. 39, no. 7, pp. e90-e92, 2016.

[20] C. Capula, E. Chiefari, M. Borelli et al., "A new predictive tool for the early risk assessment of gestational diabetes mellitus," Primary Care Diabetes, vol. 10, no. 5, pp. 315-323, 2016.

[21] A. N. Sweeting, G. P. Ross, J. Hyett et al., "Gestational diabetes mellitus in early pregnancy: evidence for poor pregnancy outcomes despite treatment," Diabetes Care, vol. 39, no. 1, pp. 75-81, 2016.

[22] L. Bozkurt, C. S. Gobl, L. Pfligl et al., "Pathophysiological characteristics and effects of obesity in women with early and late manifestation of gestational diabetes diagnosed by the International Association of Diabetes and Pregnancy Study Groups criteria," The Journal of Clinical Endocrinology \& Metabolism, vol. 100, no. 3, pp. 1113-1120, 2015.

[23] J. S. Hawkins, J. Y. Lo, B. M. Casey, D. D. McIntire, and K. J. Leveno, "Diet-treated gestational diabetes mellitus: comparison of early vs routine diagnosis," American Journal of Obstetrics and Gynecology, vol. 198, no. 3, pp. 287.e1-287.e6, 2008.

[24] J. L. Bartha, P. Martinez-Del-Fresno, and R. Comino-Delgado, "Gestational diabetes mellitus diagnosed during early pregnancy," American Journal of Obstetrics and Gynecology, vol. 182, no. 2, pp. 346-350, 2000.

[25] D. Boriboonhirunsarn and V. Kasempipatchai, "Incidence of large for gestational age infants when gestational diabetes mellitus is diagnosed early and late in pregnancy," The Journal of Obstetrics and Gynaecology Research, vol. 42, no. 3, pp. 273278, 2016.

[26] S. Easmin, T. A. Chowdhury, M. R. Islam et al., "Obstetric outcome in early and late onset gestational diabetes mellitus," Mymensingh Medical Journal, vol. 24, no. 3, pp. 450-456, 2015.

[27] J. L. Mills, L. Jovanovic, R. Knopp et al., "Physiological reduction in fasting plasma glucose concentration in the first trimester of normal pregnancy: the diabetes in early pregnancy study," Metabolism, vol. 47, no. 9, pp. 1140-1144, 1998.

[28] P. M. Catalano, E. D. Tyzbir, N. M. Roman, S. B. Amini, and E. A. Sims, "Longitudinal changes in insulin release and insulin resistance in nonobese pregnant women," American Journal of Obstetrics and Gynecology, vol. 165, no. 6, Part 1, pp. 1667-1672, 1991.

[29] E. Cosson, P. Valensi, and L. Carbillon, "Screening for dysglycaemia during pregnancy: proposals conciliating International Association of Diabetes and Pregnancy Study Group (IADPSG) and US National Institutes of Health (NIH) panels," Diabetes \& Metabolism, vol. 41, no. 3, pp. 239-243, 2015.

[30] E. Maury, J. Savel, V. Grouthier et al., "Is skin autofluorescence a marker of metabolic memory in pregnant women with diabetes?," Diabetic Medicine, vol. 32, no. 12, pp. 1575-1579, 2015.

[31] G. Rayanagoudar, A. A. Hashi, J. Zamora, K. S. Khan, G. A. Hitman, and S. Thangaratinam, "Quantification of the type 2 diabetes risk in women with gestational diabetes: a systematic review and meta-analysis of 95,750 women," Diabetologia, vol. 59, no. 7, pp. 1403-1411, 2016.

[32] E. Noctor, C. Crowe, L. A. Carmody et al., "Abnormal glucose tolerance post-gestational diabetes mellitus as defined by the International Association of Diabetes and Pregnancy Study Groups criteria," European Journal of Endocrinology, vol. 175, no. 4, pp. 287-297, 2016.

[33] K. V. Smirnakis, A. Martinez, K. H. Blatman, M. Wolf, J. L. Ecker, and R. Thadhani, "Early pregnancy insulin resistance and subsequent gestational diabetes mellitus," Diabetes Care, vol. 28, no. 5, pp. 1207-1208, 2005.

[34] C. L. Harrison, C. B. Lombard, C. East, J. Boyle, and H. J. Teede, "Risk stratification in early pregnancy for women at increased risk of gestational diabetes," Diabetes Research and Clinical Practice, vol. 107, no. 1, pp. 61-68, 2015.

[35] B. Pintaudi, G. Di Vieste, F. Corrado et al., "Improvement of selective screening strategy for gestational diabetes through a more accurate definition of high-risk groups," European Journal of Endocrinology, vol. 170, no. 1, pp. 87-93, 2014.

[36] B. Liu, H. Chen, Y. Xu et al., "Fetal growth is associated with maternal fasting plasma glucose at first prenatal visit," PLoS One, vol. 9, no. 12, article e116352, 2014.

[37] C. Wang, W. Zhu, Y. Wei et al., "The predictive effects of early pregnancy lipid profiles and fasting glucose on the risk of gestational diabetes mellitus stratified by body mass index," Journal of Diabetes Research, vol. 2016, Article ID 3013567, 8 pages, 2016.

[38] E. A. Huhn, T. Fischer, C. S. Gobl et al., "Screening of gestational diabetes mellitus in early pregnancy by oral glucose tolerance test and glycosylated fibronectin: study protocol for an international, prospective, multicentre cohort trial," $B M J$ Open, vol. 6, no. 10, article e012115, 2016.

[39] B. Liu, Y. Xu, Y. Zhang et al., "Early Diagnosis of Gestational Diabetes Mellitus (EDoGDM) study: a protocol for a prospective, longitudinal cohort study," BMJ Open, vol. 6, no. 11, article e012315, 2016.

[40] R. C. Hughes, M. P. Moore, J. E. Gullam, K. Mohamed, and J. Rowan, "An early pregnancy $\mathrm{HbA}_{1 \mathrm{c}} \geq 5.9 \%$ (41 $\left.\mathrm{mmol} / \mathrm{mol}\right)$ is optimal for detecting diabetes and identifies women at increased risk of adverse pregnancy outcomes," Diabetes Care, vol. 37, no. 11, pp. 2953-2959, 2014. 
[41] L. Mane, J. A. Flores-Le Roux, D. Benaiges et al., "Role of first trimester $\mathrm{HbAlc}$ as a predictor of adverse obstetric outcomes in a multi-ethnic cohort," The Journal of Clinical Endocrinology \& Metabolism, vol. 102, no. 2, article jc20162581, 2016.

[42] L. R. Nielsen, P. Ekbom, P. Damm et al., "HbA ${ }_{1 c}$ levels are significantly lower in early and late pregnancy," Diabetes Care, vol. 27, no. 5, pp. 1200-1201, 2004.

[43] E. Cosson, A. Benbara, I. Pharisien et al., "Diagnostic and prognostic performances over 9 years of a selective screening strategy for gestational diabetes mellitus in a cohort of 18,775 Subjects," Diabetes Care, vol. 36, pp. 598-603, 2013.

[44] V. Seshiah, A. Cynthia, V. Balaji et al., "Detection and care of women with gestational diabetes mellitus from early weeks of pregnancy results in birth weight of newborn babies appropriate for gestational age," Diabetes Research and Clinical Practice, vol. 80, no. 2, pp. 199-202, 2008.

[45] W. Y. Hong, J. R. Biggio, A. Tita, and L. M. Harper, "Impact of early screening for gestational diabetes on perinatal outcomes in high-risk women," American Journal of Perinatology, vol. 33, no. 8, pp. 758-764, 2016.

[46] A. Sweeting, F. Park, and J. Hyett, "The first trimester: prediction and prevention of the great obstetrical syndromes," Best Practice \& Research Clinical Obstetrics \& Gynaecology, vol. 29, no. 2, pp. 183-193, 2015.

[47] S. F. Ehrlich, Y. M. Crites, M. M. Hedderson, J. A. Darbinian, and A. Ferrara, "The risk of large for gestational age across increasing categories of pregnancy glycemia," American Journal of Obstetrics and Gynecology, vol. 204, no. 3, pp. 240.e1-240.e6, 2011.

[48] S. Riskin-Mashiah, A. Damti, G. Younes, and R. Auslander, "Normal fasting plasma glucose levels during pregnancy: a hospital-based study," Journal of Perinatal Medicine, vol. 39, no. 2, pp. 209-211, 2011.

[49] C. Song, J. Li, J. Leng, R. C. Ma, and X. Yang, "Lifestyle intervention can reduce the risk of gestational diabetes: a meta-analysis of randomized controlled trials," Obesity Reviews, vol. 17, no. 10, pp. 960-969, 2016. 


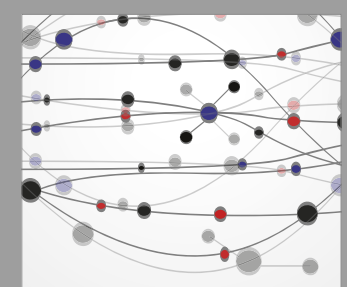

The Scientific World Journal
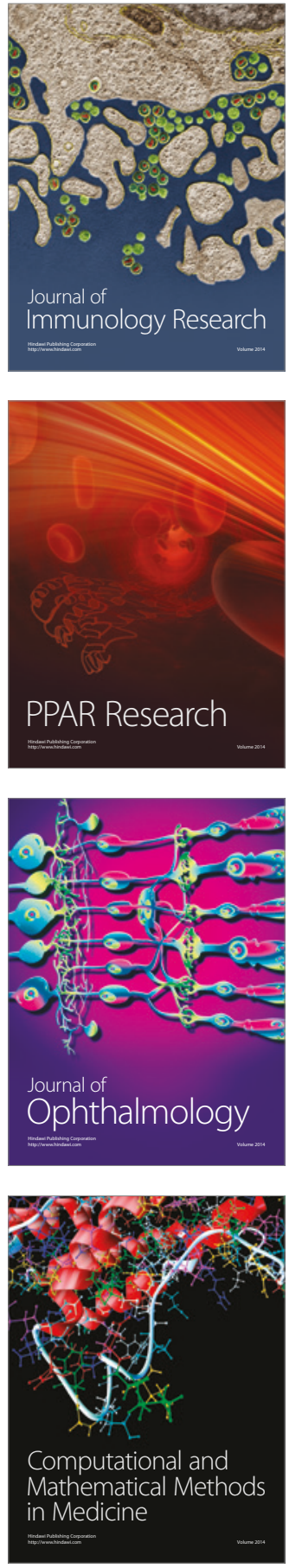

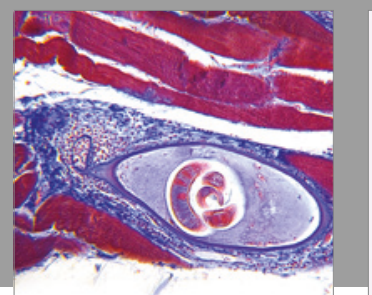

Gastroenterology Research and Practice
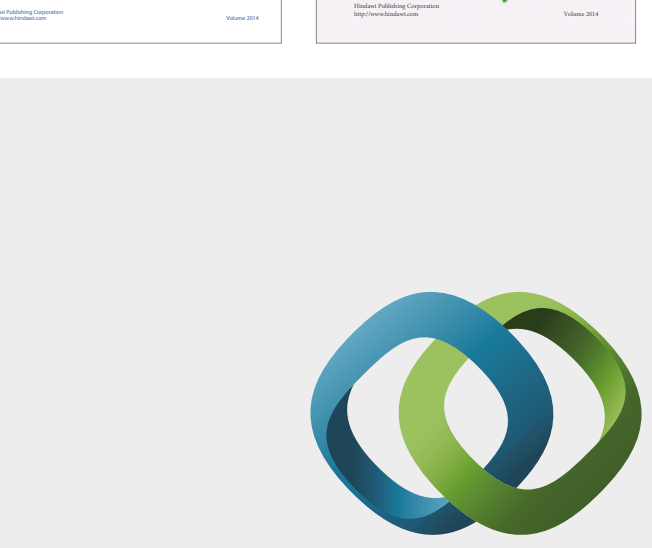

\section{Hindawi}

Submit your manuscripts at

https://www.hindawi.com
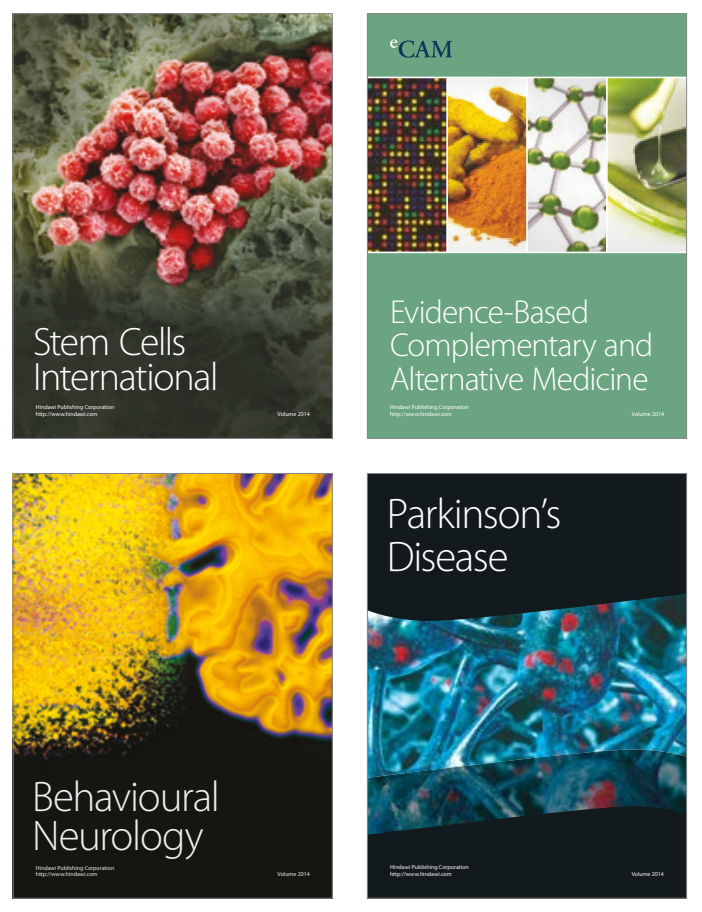
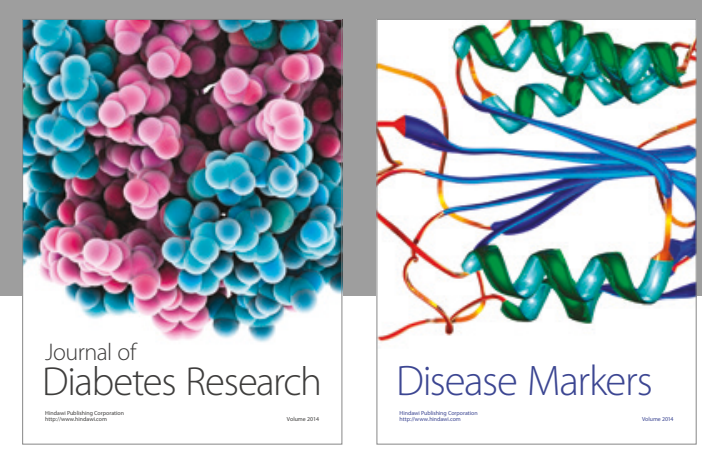

Disease Markers
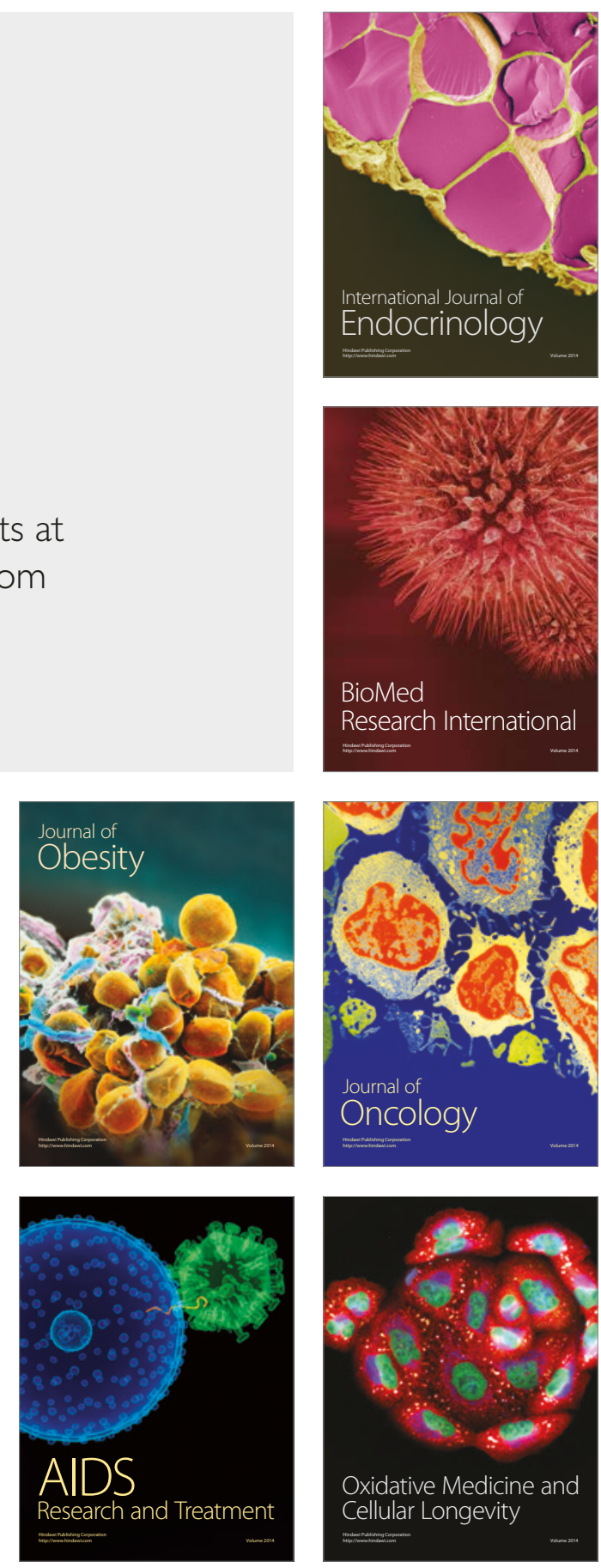